\title{
Predictive role of UCA1-containing exosomes in cetuximab-resistant colorectal cancer
}

Ying-nan Yang ${ }^{1 \dagger}$, Rui Zhang ${ }^{2 \dagger}$, Jing-wen Du ${ }^{3}$, Heng-heng Yuan ${ }^{3}$, Yan-jing Li ${ }^{3}$, Xiao-li Wei ${ }^{3}$, Xiao-xue Du ${ }^{3}$, Shu-lin Jiang ${ }^{4^{*}}$ and Yu $\operatorname{Han}^{3^{*}}$ (B)

\begin{abstract}
Background: Primary or acquired resistance to cetuximab often occurs during targeted therapy in metastatic colorectal cancer (mCRC) patients. In many cancers, the key role of the long noncoding RNA (IncRNA) urothelial carcinoma-associated 1 (UCA1) in anticancer drug resistance has been confirmed. Emerging evidence has shown that specific exosomal IncRNAs may serve as meaningful biomarkers. In this study, we hypothesize that exosomal UCA1 might predict the response to cetuximab in CRC patients.
\end{abstract}

Methods: First, acquired cetuximab-resistant cell lines were generated, and UCA1 expressions in these cells and their exosomes were compared. We also systematically evaluate the stability of exosomal UCA1. Thereafter, the predictive value of exosomal UCA1 in CRC patients treated with cetuximab was evaluated. Finally, through cell apoptosis assays and immunofluorescence staining, we analyzed the role of UCA1-containing exosomes in conferring cetuximab resistance.

Results: UCA1 expression was markedly higher in cetuximab-resistant cancer cells and their exosomes. Exosomal UCA1 was shown to be detectable and stable in serum from CRC patients. In addition, circulating UCA1-containing exosomes could predict the clinical outcome of cetuximab therapy in CRC patients, and UCA1 expression was considerably higher in the progressive disease/stable disease patients than in the partial response/complete response patients. Furthermore, exosomes derived from cetuximab-resistant cells could alter UCA1 expression and transmit cetuximab resistance to sensitive cells.

Conclusions: We discovered a novel role of UCA1-containing exosomes, showed their capability to transmit drug resistance and investigated their potential clinical use in predicting cetuximab resistance.

Keywords: UCA1, Exosomes, Cetuximab, Metastatic colorectal cancer, Biomarker

\section{Background}

Colorectal cancer (CRC) is a leading cause of cancer-related death worldwide [1]. Screening, surgery, and medical therapies are usually effective for the

\footnotetext{
*Correspondence: sljiang36@163.com; hanyu_2017@126.com

†Ying-nan Yang and Rui Zhang contributed equally to this work

${ }^{3}$ Department of Gastrointestinal Oncology, Harbin Medical University

Cancer Hospital, 150 \# Haping Road, Harbin 150081, Heilongjiang, China

${ }^{4}$ Department of Cardiovascular Surgery, The Second Affiliated Hospital

of Harbin Medical University, 246 \# Xuefu Road, Harbin 150001,

Heilongjiang, China

Full list of author information is available at the end of the article
}

management of early-stage CRC, however, these treatment options are far less effective for advanced-stage disease. Cetuximab is an epidermal growth factor receptor (EGFR) monoclonal antibody (mAb) that binds the EGFR extracellular domain and enhances receptor internalization and degradation. This mAb targeting EGFR is a common targeted agent used to treat patients with metastatic CRC (mCRC) with wild-type KRAS status [2]. However, patients frequently develop drug resistance. A number of genetic biomarkers, including RAS (KRAS exons 3 and 4 or NRAS exons 2,3 , and 4) mutations [3, 4], BRAF (V600E) [5], PIK3CA (exon 20) [6] and HER2 and 
MET amplification [7, 8], are robustly correlated with cetuximab resistance. Despite these genetic biomarkers, additional mechanisms of resistance to EGFR mAb are thought to be present in mCRC.

Currently, with advancements in global transcriptome profiling technique, the functional roles of long noncoding RNAs (lncRNAs) have received considerable attention in human cancer [9]. LncRNAs are mRNA-like transcripts ranging in length from 200 nucleotides (nt) to over 100 kilobases $(\mathrm{kb})$ lacking significant protein-coding ability [10]. Emerging evidence has demonstrated that lncRNAs play significant roles in biological processing, including cell cycle regulation, apoptosis and tumor invasion [11]. Additionally, other lncRNAs have been shown to play functional roles in resistance to targeted therapy [12]. Some lncRNAs could be potentially used as early diagnostic, prognostic and drug response biomarkers in malignant tumors [12-14].

Urothelial carcinoma-associated 1 (UCA1) is a lncRNA with three exons, and several recent studies have demonstrated oncogenic functions of UCA1 in various types of cancer, such as breast, bladder, colorectal, and gastric cancer [15]. In our previous study, we elucidated that UCA1 expressions are significantly increased in CRC tissues and cells, and this high UCA1 expression level is significantly correlated with larger tumor size, greater tumor depth and less differentiated histology. Additionally, patients with high UCA1 level have a significantly poorer prognosis than those with low UCA1 level. Moreover, UCA1 influences the proliferation, cell cycle and apoptosis of CRC cells. These data indicate an important role for UCA1 in the molecular etiology of CRC and implicate a potential application for UCA1 in CRC diagnosis, prognosis of tumor progression, and therapy [16]. In addition to its oncogenic function, UCA1 regulates drug resistance in some kinds of malignant tumors [17]. However, the value of UCA1 levels in body fluid samples regarding the response of CRC patients to cetuximab remains to be confirmed.

Exosomes are lipid vesicles with a diameter of $30-100 \mathrm{~nm}$ that are secreted by the fusion of multivesicular bodies with the plasma membrane or by budding from the membrane [18]. Nucleic acids, proteins and lipids are loaded in exosomes, thereby allowing the transfer of genetic material and enabling the exchange of information between cells. Emerging evidence has demonstrated that some specific exosomal lncRNAs expression is correlated with cancer patients' clinicopathological characteristics and therefore may act as a potential biomarker [18-20]. Based on these observations, we hypothesize that exosomal UCA1 might predict the response of CRC patients to cetuximab.
The existence and stability of exosomal UCA 1 in serum were systematically investigated in this study. Additionally, we identified a novel role of UCA1-containing exosomes in predicting the response of CRC patients to cetuximab. Moreover, for the first time, we suggest that UCA1 may be transmitted via exosomes, thus affecting drug metabolism.

\section{Methods}

\section{Patients and sample processing}

Informed consent was obtained from each patient, and the protocols were approved by the Ethics Committee of Harbin Medical University Cancer Hospital. Serum samples were collected from Harbin Medical University Cancer Hospital to investigate the feasibility of detecting exosomal UCA1. Changes in serum exosomal UCA1 levels were validated in an independent cohort of 53 CRC patients treated with cetuximab at Harbin Medical University Cancer Hospital from 2015 to 2016. Blood samples from all CRC patients were collected by vena puncture. To extract exosome from human blood, a two-step centrifugation protocol $-1600 \mathrm{~g}$ for $10 \mathrm{~min}$ and $16,000 \mathrm{~g}$ for $10 \mathrm{~min}$ at $4{ }^{\circ} \mathrm{C}$ was used to isolate serum, and then serum was stored at $-80{ }^{\circ} \mathrm{C}$ until exosome extraction. Blood samples with evidence of hemolysis were excluded. According to the RECIST criteria for a pathological response, these patients were divided into two groups: 30 patients responded to cetuximab therapy [complete response $(\mathrm{CR})$ or partial response (PR)], and 23 patients did not respond [stable disease (SD) or progressive disease (PD)].

\section{Cell lines and culture}

The human Caco2 cell line was purchased from the Cell Bank of the Chinese Academy of Sciences (Shanghai, China). We established cetuximab-resistant cell lines by chronically exposing cetuximab-sensitive Caco2 (Caco2CS) cells to increasing cetuximab doses in medium over a period of 6 months. The final concentration of cetuximab for the cetuximab-resistant subclone Caco2-CR was $300 \mu \mathrm{g} / \mathrm{ml}$. Caco2-CS and Caco2-CR cells were cultured in Dulbecco's modified Eagle's medium (DMEM, Gibco, Invitrogen, USA) containing $10 \%$ fetal bovine serum (FBS, Gibco, Invitrogen, USA) and $1 \%$ penicillin-streptomycin (Invitrogen, USA) at $37{ }^{\circ} \mathrm{C}$ in a humidified atmosphere of $95 \%$ air $/ 5 \% \mathrm{CO}_{2}$.

\section{Cell proliferation assay and colony formation assay}

For the cell proliferation assay, cell viability was determined by Cell Counting Kit 8 (CCK8, Dojindo, Japan) according to the manufacturer's instructions. For the colony formation assay, about 1000 cells were placed in each well of a 6 -well plate in $2 \mathrm{ml}$ media containing 
cetuximab $(300 \mu \mathrm{g} / \mathrm{ml}$ for Caco2-CR). The media were changed every 3 day. After 12-15 days, the colonies were fixed in $80 \%$ methanol and stained with $0.1 \%$ crystal violet for $20 \mathrm{~min}$. The number of colonies was counted using an inverted microscope.

\section{Isolation of exosomes}

Medium and serum were filtered through a $0.45 \mu \mathrm{m}$ polyvinylidene fluoride filter (Millipore, Billerica, MA, USA); ExoQuick solution (System Biosciences, Mountain View, CA, USA) was added to the serum, which was then incubated for $0.5 \mathrm{~h}$ at room temperature, and ExoQuick-TC solution was added to the medium, which was then incubated at $4{ }^{\circ} \mathrm{C}$ for $12 \mathrm{~h}$. The mixture was centrifuged at $1500 \mathrm{~g}$ for $30 \mathrm{~min}$ and supernatant was removed by aspiration. Pelleted fractions were resuspended in $25 \mu \mathrm{l}$ phosphate-buffered saline (PBS).

\section{Transmission electron microscopy (TEM)}

A sample of exosomes was diluted to a final concentration of $0.5 \mathrm{mg} / \mathrm{ml}$ in PBS, spotted onto a glow-discharged copper grid on filter paper and dried for $10 \mathrm{~min}$. Exosomes were stained with $1 \%$ aqueous phosphotungstic acid (PTA) for $5 \mathrm{~min}$ and dried for $20 \mathrm{~min}$ and then examined at $100 \mathrm{keV}$ with TEM (JEM-1-11 microscope, Japan).

\section{RNA extraction}

Total RNA was extracted from cells using TRIzol ${ }^{\circledR}$ Reagent (Invitrogen, CA, USA). Exosomal RNA was extracted using the Total Exosome RNA and Protein Isolation Kit (Invitrogen, USA). The concentration and quality of RNA were measured by UV absorbance at 260 and $280 \mathrm{~nm}(260 / 280 \mathrm{~nm})$ using a Nanodrop 2000 spectrophotometer (Thermo Scientific, USA).

\section{Quantitative real-time reverse transcription-polymerase chain reaction (qRT-PCR)}

Total RNA was extracted from cells and exosomes as described above. RNA templates were treated with DNase I to avoid genomic DNA contamination. The first strand of cDNA was synthesized using the SuperScript First-Strand Synthesis System (Invitrogen, CA). PCR amplification was performed using an Applied Biosystems 7500 Detection System (Applied Biosystems, CA) and primers for UCA1 (forward: $5^{\prime}$-ACGCTAACTGGC ACCTTGTT-3', reverse: 5'-TGGGGATTACTGGGG TAGGG-3') and $\beta$-actin (forward, 5'-CACCTTCTA CAATGAGCTGCGTGTG-3'; reverse, 5'-ATAGCACAG CCTGGATAGCAACGTAC-3'). Real-time PCR was performed on triplicate samples according to the instructions of the SYBR ${ }^{\circledR}$ Premix Ex Taq ${ }^{\mathrm{TM}}$ Kit (Takara, Japan). The expression level of UCA1 was normalized to that of $\beta$-actin using the comparative $2^{-\Delta \Delta \mathrm{Ct}}$ method.

\section{Western blot analysis}

Proteins were extracted from cells using RIPA lysis buffer (Biouniquer Technology, China). Exosomal proteins were extracted using the Total Exosome RNA and Protein Isolation Kit (Invitrogen, USA). Protein content was measured using a Nanodrop 2000 spectrophotometer (Thermo Scientific, USA). Equal amounts of protein from each sample were separated by electrophoresis in sodium dodecyl sulfate (SDS)-polyacrylamide gels before being transferred onto polyvinylidene difluoride (PVDF) membranes, blocked for $2 \mathrm{~h}$ with $5 \%$ skim milk, incubated overnight at $4{ }^{\circ} \mathrm{C}$ with primary antibodies against TSG101, Alix and CD81 (Abcam, Cambridge, UK) and then incubated for $1 \mathrm{~h}$ with the secondary antibody (Kangwei Ltd., China). The signals were visualized with an ECL detection system.

\section{Immunofluorescence assay}

For exosomal labeling, Dil (Beyotime Biotechnology, China) was added to the exosome suspension at $1 \mu \mathrm{M}$ for $20 \mathrm{~min}$, and the exosome suspension was then washed through Exosome Spin Columns (MW3000) (Invitrogen, USA) to remove excess dye. Dil-labeled exosomes were incubated with CRC cells and visualized by fluorescence microscopy or EPICS XL flow cytometry (Beckman Coulter) after $24 \mathrm{~h}$.

\section{RNA interference}

Caco2-CR cells were transfected with UCA1-small interfering RNA (siRNA) (RNAi: forward primer: 5'-GCA CCUUGUUAGCUACAUAAA- ${ }^{\prime}$, reverse primer: 5'-UAUGUAGCUAACAAGGUGCCA- $3^{\prime}$ ) using Lipofectamine $^{\mathrm{TM}}$ 2000. After $48 \mathrm{~h}$ of transfection, RNAi efficiency was assessed by qRT-PCR. Another siRNA was designed as a negative control (Ctrl siRNA): forward primer: $\quad 5^{\prime}$-UUCUCCGAACGUGUCACGUdTdT3', reverse primer: 5'-ACGUGACACGUUCGGAGA AdTdT-3'.

\section{Electroporation of UCA1 into exosomes}

UCA1 was electroporated into exosomes using a GenePulser $\mathrm{Xcell}^{\mathrm{TM}}$ electroporation system (BioRad, USA) as previously described [21]. Briefly, $2 \mu \mathrm{g}$ exosomes and $400 \mathrm{nmol}$ RNA were mixed in $400 \mu \mathrm{l}$ electroporation buffer and electroporated at $350 \mathrm{~V}$ and $150 \mu \mathrm{F}$ in a 4-mm cuvette. The mixture was incubated at $37{ }^{\circ} \mathrm{C}$ for $30 \mathrm{~min}$ to ensure that the exosome membrane fully recovered, and then, the mixture was treated with RNase to remove unincorporated RNA. Labeled RNA in exosomes was quantified by detecting fluorescence using a Fluorescence Spectrophotometer F-4600 (HITACHI, Japan). 


\section{Apoptosis assay}

Twenty-four hours after $1 \times 10^{5}$ Caco2-CS cells were seeded in six-well plates, different amounts of CRexo $(1,5$, or $10 \mu \mathrm{g})$ were added to the supernatants. The samples were divided into the following four groups: CS, CS $+1 \mu$ g CR-exo, CS $+5 \mu \mathrm{g}$ CR-exo, and $\mathrm{CS}+10 \mu \mathrm{g}$ CR-exo. After culturing for $24 \mathrm{~h}$, the cells were treated with cetuximab $(300 \mu \mathrm{g} / \mathrm{ml})$ for $48 \mathrm{~h}$. Then, all the cells were harvested by centrifugation, washed with PBS, stained using Annexin V fluorescein isothiocyanate (APC) and propidium iodide (PI) and analyzed using a flow cytometer (BD Biosciences, San Diego, CA).

\section{Statistical analyses}

All statistical analyses in this study were performed with SPSS 16.0 software. All experiments were carried out three times independently. Data are presented as the mean \pm standard deviation. $\mathrm{p}$ values $<0.05$ by Student's t-test or one-way ANOVA indicated statistical significance. All statistical tests were two-sided.

\section{Results}

Upregulation of UCA1 levels in cetuximab-resistant cells and their exosomes

We generated cetuximab-resistant Caco2-CR cells from Caco2-CS CRC cells following long-term exposure to increasing doses of cetuximab. Caco2-CR cell lines could grow in culture medium containing $300 \mu \mathrm{g} / \mathrm{ml}$ cetuximab and displayed a robust cetuximab-resistant phenotype as compared to parental controls (Fig. 1a). Additionally, the clonogenicity of Caco2-CR cells was significantly greater than that of Caco2-CS cells in response to cetuximab treatment (Fig. 1b).

Total RNA was extracted from Caco2-CS and Caco2CR cells, and UCA1 expression levels were assessed using qRT-PCR. UCA1 expression was markedly higher in Caco2-CR cells than in parental Caco2-CS cells (Fig. 1c). We then isolated exosomes from the conditioned medium of Caco2-CS and Caco2-CR cells. Western blot analysis demonstrated the expression of TSG101, Alix, and CD81, which are all exosome markers and are associated with exosome formation, in both exosomes and cells (Fig. 1d). In addition, TEM revealed that the predominant vesicles were of typical exosome size (30-150 $\mathrm{nm}$ in diameter), had a characteristic round or saucer shape, and were delineated by a lipid bilayer (Fig. 1e). Further quantitative analysis suggested that the number of exosomes expelled from both cell variants did not differ significantly ( $p>0.05$; Fig. 1f). Moreover, we detected UCA1 levels in exosomes derived from Caco2CR cells (CR-exo) and Caco2-CS cells (CS-exo) and found that UCA1 levels were significantly upregulated in CR-exo compared with CS-exo (Fig. 1g). Interestingly, the increase in UCA1 levels between CR-exo and CS-exo

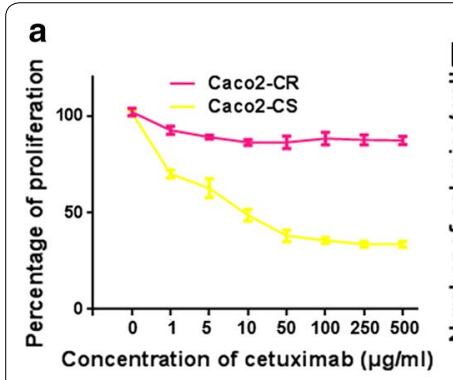

e

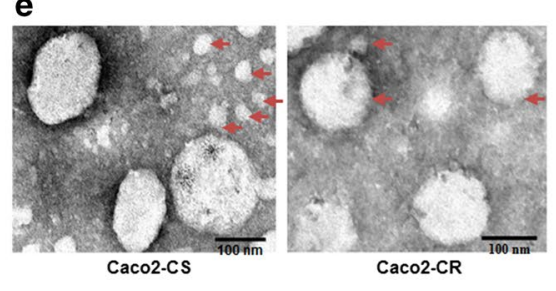

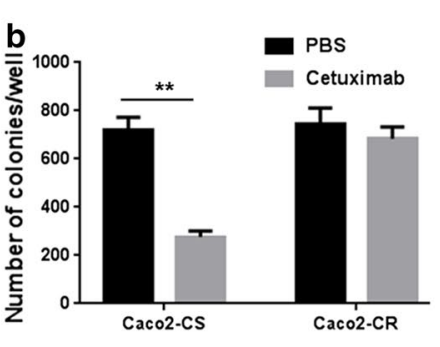

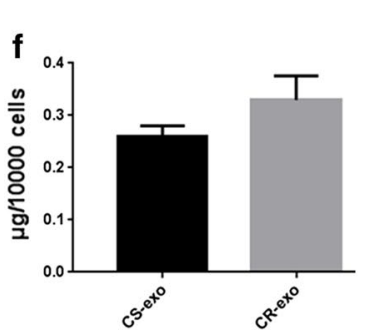

d
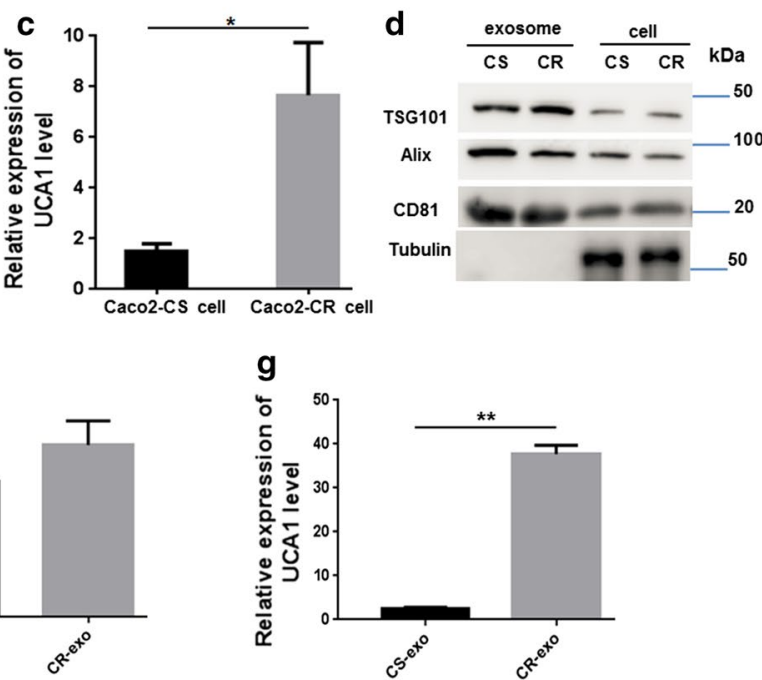

Fig. 1 Levels of UCA1 in cetuximab-resistant and cetuximab-sensitive cells and their exosomes. a We treated Caco2-CS and Caco2-CR cells with increased concentrations of cetuximab and evaluated the cell proliferation by CCK8 assay. $\mathbf{b}$ Colony formation assays were performed to determine the effect of PBS or cetuximab $(300 \mu \mathrm{g} / \mathrm{ml})$ on Caco2-CS and Caco2-CR cells colony formation. ${ }^{* *} \mathrm{p}<0.01$. c UCA1 levels were significantly higher in Caco2-CR cells than in Caco2-CS cells; ${ }^{*} p<0.05$. Data are shown as the mean \pm SD. $\mathbf{d}$ Western blot analyses were used to detect exosome markers (CD81 and TSG101) in Caco2-CS (CS) cells and Caco2-CR (CR) cells. e Exosomes were observed by TEM. Exosomes are highlighted using red arrows. Scale bar, $100 \mathrm{~nm}$. f Quantification of exosomes expelled from equal numbers of Caco2-CS/CR cells. $\mathbf{g}$ UCA1 levels were higher in CR-exo than in CS-exo; ${ }^{* *} \mathrm{p}<0.01$ 
( 20-fold) was much greater than that between Caco2$\mathrm{CR}$ and Caco2-CS cells ( $\sim$-fold), indicating that UCA1 was concentrated in exosomes derived from Caco2-CR cells; moreover, UCA1 expressions may be related to cetuximab resistance in CRC cells.

\section{Characterization of serum exosomal UCA1}

Figure 2a shows the relative expression of lncRNA UCA1 acquired using the same amount of total RNA. qRTPCR analysis indicated that the exosomal UCA1 could be reliably detected but outside exosomal UCA1 was only minimally detected. To explore the potential benefit of detecting exosomal UCA1, we skipped the exosome extraction step and extracted RNA from whole serum directly. The data presented in Fig. 2a suggested that UCA1 levels were higher in exosome samples than in whole serum samples $(\mathrm{p}<0.05)$.

To ascertain whether the exosomal UCA1 can be protected by exosome membrane, exosome samples and nucleic acids isolated from exosome were subjected to harsh conditions. Samples were incubated with RNase $\mathrm{A}$, a strong acid, or a strong base for $3 \mathrm{~h}$ at room temperature, and the results indicated that RNase A (Fig. 2b) and acid or base (Fig. 2c) treatments had minimal effects on the expression of exosomal UCA1 in the exosome group but caused complete degradation of UCA1 in the isolated nucleic acid group. In the freeze-thaw cycle test, exosomal UCA1 levels showed no significant change but decreased markedly in the isolated nucleic acid group after eight freeze-thaw cycles (Fig. 2d). The levels of exosomal UCA1 in the exosome group were not significantly changed from 0 to $36 \mathrm{~h}$ at room temperature, but were markedly decreased in the isolated nucleic acid group after $24 \mathrm{~h}$ (Fig. 2e). Moreover, as shown in Fig. 2f, exosomal UCA1 remained stable in both groups at $-80{ }^{\circ} \mathrm{C}$. Collectively, these data suggest that exosomal UCA1 is detectable and stable in exosomes, providing a basis for its use as a predictive biomarker of the response to cetuximab.

\section{Circulating UCA1-containing exosomes predict the clinical outcome of cetuximab therapy in CRC patients}

In order to investigate whether UCA1-containing exosomes could be a useful tumor-derived material for predicting the clinical outcome of cetuximab therapy in CRC patients, we used qRT-PCR to evaluate the features of UCA1-containing exosomes in the peripheral blood from 53 patients who were treated with cetuximab in combination with chemotherapy either as first-line $(\mathrm{n}=39,73.6 \%)$ or third-line therapy $(n=14,26.4 \%)$. The main characteristics of all enrolled patients are summarized in Table 1. All subjects were confirmed to have wild-type KRAS (exon 2) before cetuximab administration. Patient' ages ranged from 31 to 82 years. Tumors were staged according to

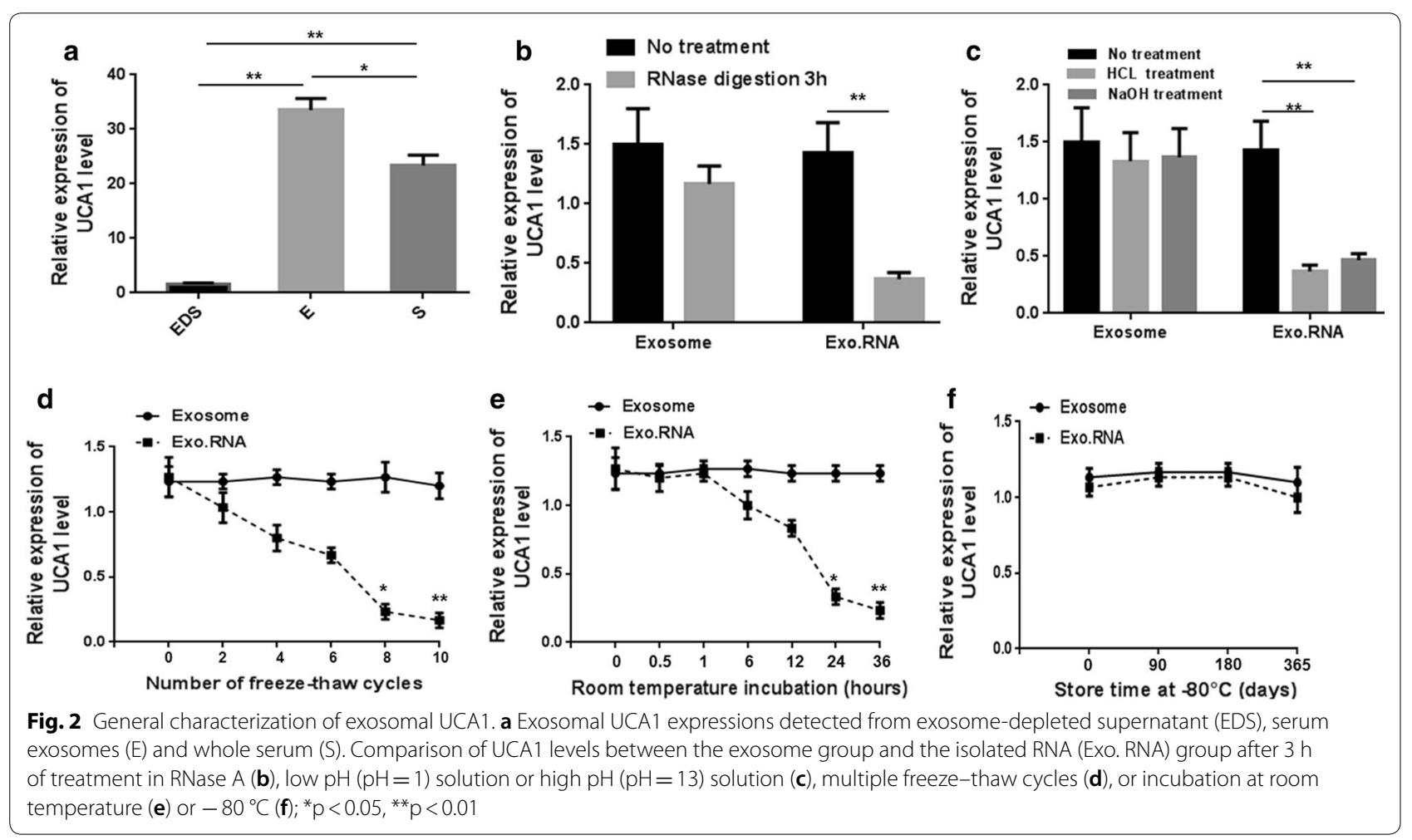


the American Joint Committee on Cancer (AJCC) 2010 guidelines. Based on patient response to cetuximab, the cohort was separated into two groups: the responder group included $30(56.6 \%)$ patients with either a $\mathrm{CR}(\mathrm{n}=3$, $5.7 \%)$ or PR $(\mathrm{n}=27,50.9 \%)$, and the nonresponder group included $23(43.4 \%)$ patients with SD $(n=17,32.1 \%)$ or PD $(n=6,11.3 \%)$. Similar response rates were found among the first-line-treated patients $(59.0 \%, 23$ of 39) and the third-line-treated patients ( $50.0 \%, 7$ of 14 ).

As shown in Fig. 3a, in equal amounts of serum, the quantity of exosomes was higher (1.3-fold) in patients who did not respond to cetuximab therapy (PD/SD) than in patients who responded to treatment (PR/CR), but

\section{Table 1 Characteristics of patients with metastatic colorectal cancer}

\begin{tabular}{|c|c|c|c|c|}
\hline & $\begin{array}{l}\text { All patients } \\
\mathrm{n}(\%)\end{array}$ & $\begin{array}{l}\text { Responders } \\
\mathrm{n}(\%)\end{array}$ & $\begin{array}{l}\text { Non- } \\
\text { responders } \\
\mathrm{n}(\%)\end{array}$ & $p$-value* \\
\hline Total number & $53(100)$ & $30(56.6)$ & $23(43.4)$ & \\
\hline \multicolumn{4}{|l|}{ Sex } & \multirow[t]{3}{*}{ NS } \\
\hline Male & $31(58.5)$ & $18(60.0)$ & $13(56.5)$ & \\
\hline Female & $22(41.5)$ & $12(40.0)$ & $10(43.5)$ & \\
\hline \multicolumn{4}{|l|}{ Age } & \multirow[t]{4}{*}{ NS } \\
\hline$\leq 70$ & $38(71.7)$ & $25(83.3)$ & $13(56.5)$ & \\
\hline$>70$ & $15(28.3)$ & $5(16.7)$ & $10(43.5)$ & \\
\hline Median (range) & $56(31-82)$ & $51.5(31-82)$ & $59(37-77)$ & \\
\hline \multicolumn{4}{|c|}{ Histologic grade } & \multirow[t]{3}{*}{ NS } \\
\hline Low grade ${ }^{a}$ & $45(84.9)$ & $27(90.0)$ & $18(78.3)$ & \\
\hline High grade $^{b}$ & $8(15.1)$ & $3(10.0)$ & $5(21.7)$ & \\
\hline \multicolumn{4}{|c|}{ Metastatic pattern } & \multirow[t]{3}{*}{ NS } \\
\hline $\begin{array}{l}\text { Metachro- } \\
\text { nous }\end{array}$ & $14(26.4)$ & $7(23.3)$ & $7(30.4)$ & \\
\hline Synchronous & $39(73.6)$ & $23(76.7)$ & $16(69.6)$ & \\
\hline \multicolumn{4}{|c|}{ Primary tumor site } & \multirow[t]{3}{*}{ NS } \\
\hline Colon & $32(60.4)$ & $18(60.0)$ & $14(60.9)$ & \\
\hline Rectum & $21(39.6)$ & $12(40.0)$ & $9(39.1)$ & \\
\hline \multicolumn{5}{|l|}{ Metastatic site } \\
\hline Liver & 37 & 20 & 17 & \multirow[t]{3}{*}{ NS } \\
\hline Lung & 21 & 11 & 10 & \\
\hline Other & 22 & 9 & 13 & \\
\hline \multicolumn{4}{|c|}{ Number of metastatic site } & \multirow[t]{3}{*}{ NS } \\
\hline 1 & $30(56.7)$ & $19(63.3)$ & $11(47.8)$ & \\
\hline$>1$ & $23(43.4)$ & $11(36.7)$ & $12(52.2)$ & \\
\hline \multicolumn{4}{|c|}{ Treatment regimen } & \multirow[t]{3}{*}{ NS } \\
\hline First line & $39(73.6)$ & $23(76.7)$ & $16(69.6)$ & \\
\hline Third line & $14(26.4)$ & $7(23.3)$ & $7(30.4)$ & \\
\hline
\end{tabular}

NS not significant

* Fisher exact $p$-value

a Well-differentiated/moderately-differentiated

b Poorly-differentiated this difference was not significant $(\mathrm{p}>0.05)$. Interestingly, serum exosomal UCA1 expression was significantly higher in the $\mathrm{PD} / \mathrm{SD}$ group than in the PR/CR group $(\mathrm{p}<0.01$; Fig. $3 \mathrm{~b}$ ), suggesting that high exosomal UCA1 expression levels might predict increased resistance to cetuximab therapy.

\section{UCA1 can be transferred from resistant cells to sensitive cells through exosomes}

Previous studies have suggested that UCA1 is a resistance factor for anticancer drugs [17]. In addition, cell-secreted exosomes and capped nucleic acid cargo can be internalized by neighboring cells $[22,23]$. Accordingly, we reasoned that exosomes may carry UCA1 and mediate its intercellular transport. First, CR-exo were labeled with the dye PKH26 and then added to the culture medium of Caco2CS cells in order to investigate whether exosomes are internalized by recipient cells. Microscopic images showed that labeled exosomes were internalized by Caco2-CS cells in a time-dependent manner after coincubation (Fig. 4a). To further confirm that UCA1 could be transferred to recipient cells via exosomes, Caco2-CS cells were electroporated with fluorescein isothiocyanate (FITC)-UCA1, then exosomes were isolated and labeled with Dil. As shown in Fig. 4b, we observed that colocalization of FITC and Dil was in most recipient cells, whereas no internalization of naked FITC-UCA1 was found in recipient cells after incubation with labeled exosomes. Moreover, to evaluate whether the transport of exosomes derived from drugresistant cells altered UCA1 levels, we coincubated Caco2CS cells with CS-exo or CR-exo for $48 \mathrm{~h}$, and UCA1 levels in the Caco2-CS, Caco2-CS + CS-exo, Caco2-CS + CR-exo and Caco2-CR groups were analyzed by qRT-PCR. The level of UCA1 increased in the Caco2-CS + CR-exo group compared with the Caco2-CS and Caco2-CS+CS-exo groups but did not increase in UCA1-knockdown-resistant cells; however, UCA1 levels did not significantly change in the Caco2-CS + CS-exo group compared with the Caco2CS group (Fig. 4c). These results show that exosomes carrying UCA1 content from cetuximab-resistant cells can be transferred to recipient sensitive cells.

\section{Exosomes derived from cetuximab-resistant cells can transmit drug resistance to sensitive cells and alter UCA1 expression}

In the end, we investigated the role of UCA1-containing exosomes in the transfer of cetuximab resistance. We added different amounts of CR-exo to the medium of Caco2-CS cells (CS $+1 / 5 / 10 \mu \mathrm{g}$ CR-exo, Caco2-CS cells were used as the control group). After $24 \mathrm{~h}$ of culture, we added serial dilutions of cetuximab to all groups. Two days later, we found a reduction in apoptosis in the groups coincubated with CR-exo $(\mathrm{CS}+1 / 5 / 10 \mu \mathrm{g}$ 

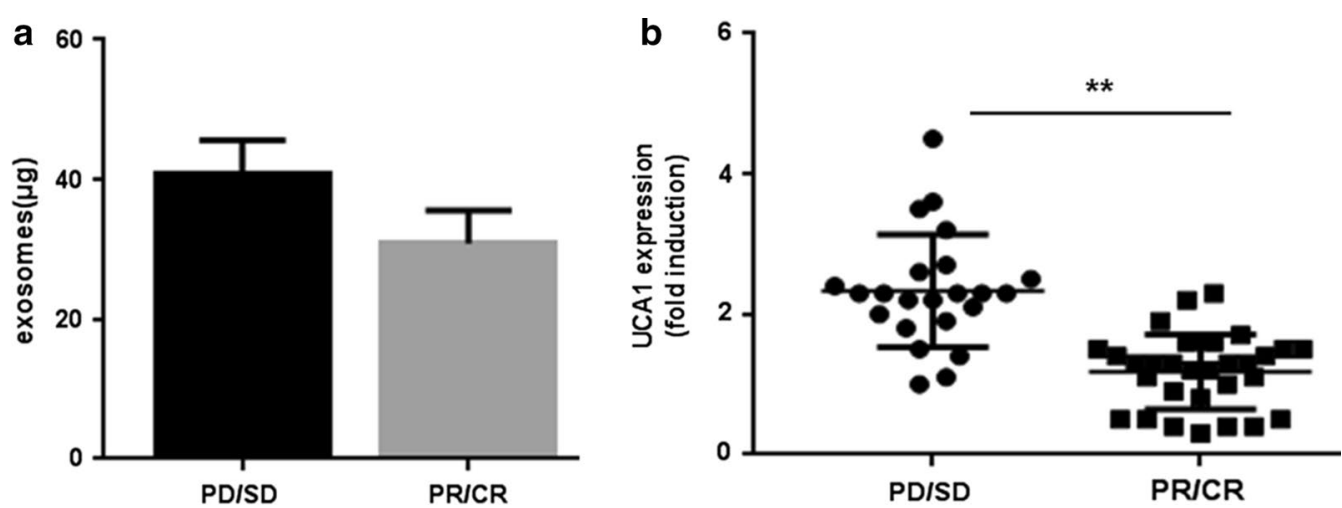

Fig. 3 Circulating UCA1-containing exosomes predict the clinical outcome of cetuximab therapy in CRC patients. a The quantity of exosomes was higher in PD/SD patients $(n=23)$ than in PR/CR patients $(n=30)$, but this difference was not significant. $\mathbf{b}$ qRT-PCR assays showed a higher UCA1 expression in circulating serum exosomes in PD/SD patients than in those in PR/CR patients $(p<0.01)$

CR-exo) compared with the CS group $(\mathrm{p}<0.05)$, indicating the increased resistance to cetuximab (Fig. 5a). Interestingly, the apoptosis rates of Caco2-CS cells incubated with CR-exo were inversely dependent on the CR-exo dose. Moreover, UCA1 levels significantly increased in CR-exo-supplemented cells in a dose-dependent manner (Fig. 5b). Thus, we reasoned that UCA1 might play a role in cetuximab resistance and that exosomal UCA1 might transmit cetuximab resistance in CRC cells, resulting in greater cetuximab resistance in recipient cells.

\section{Discussion}

Cetuximab is one of the most widely used EGFR inhibitors in the treatment of mCRC patients with wild-type KRAS status. However, primary or acquired resistance to cetuximab often occurs during targeted therapy. To optimize individualized cetuximab therapy in CRC patients, it is essential to identify possible indicators of the response to cetuximab therapy.

The IncRNA UCA1 has been found to reactive and is a promising diagnostic and prognostic biomarker in various malignant tumors [15]. Furthermore, many studies have shown that UCA1 induces drug resistance in various cancers [17]. The role of UCA1 in CRC drug resistance has also been described [24]. Bian et al. [24] showed that ectopic expression of UCA1 increased the chemoresistance of CRC cells to 5-fluorouracil (5-FU), whereas knockdown of UCA1 enhanced apoptosis in HCT116 cells. Mechanistically, UCA1 acts as a sponge for miR-204-5p and upregulates the level of several target genes of miR-204-5p, suggesting the important role of UCA1 in chemoresistance. Therapeutic monitoring using human blood is considered a noninvasive and convenient method, and circulating lncRNAs as biomarkers for predicting therapeutic efficacy in cancer has been investigated for years $[19,25]$. However, the potential of using UCA1 levels in body fluid samples as a predictive biomarker of drug resistance remains to be confirmed.

Increasing evidence indicates that the tumor microenvironment can also contributes to changes in tumor characteristics [26, 27], such as resistance to some anticancer drugs $[28,29]$. Exosomes are a promising tumorderived material for the characterization of some tumor behaviors [30, 31]. Sugimachi et al. [32] showed that serum exosomal miRNAs could be potential novel biomarkers for predicting tumor recurrence and prognosis. Furthermore, Ma et al. [33] demonstrated that TrpC5-containing extracellular vesicles might be a potential biomarker for chemoresistant breast cancer. Here, we sought to investigate whether exosomal UCA1 is involved in the drug resistance process and to determine the significance of the transfer of cetuximab-resistance properties in CRC. In the current study, we first clarified the upregulation of UCA1 levels in cetuximab-resistant cells and their exosomes. Interestingly, it was observed that UCA1 detectable in cells and serum is concentrated in exosomes. In fact, the increase in exosomes is more evident than in cells, and therefore, purifying exosomes could improve the sensitivity of circulating UCA1 detection. Therefore, we reason that exosome-mediated transfer of UCA1 might be an important mechanism of acquitted cetuximab resistance in CRC cells. We also systematically evaluated the stability of exosomal UCA1, and the results indicated that exosomal UCA1 is evidently stable when subjected to severe conditions, suggesting that the exosomal UCA1 can be protected by exosome membrane. Furthermore, we observed that circulating UCA1-containing exosomes could predict the clinical outcome of cetuximab therapy in CRC patients. Although the quantity of exosomes 

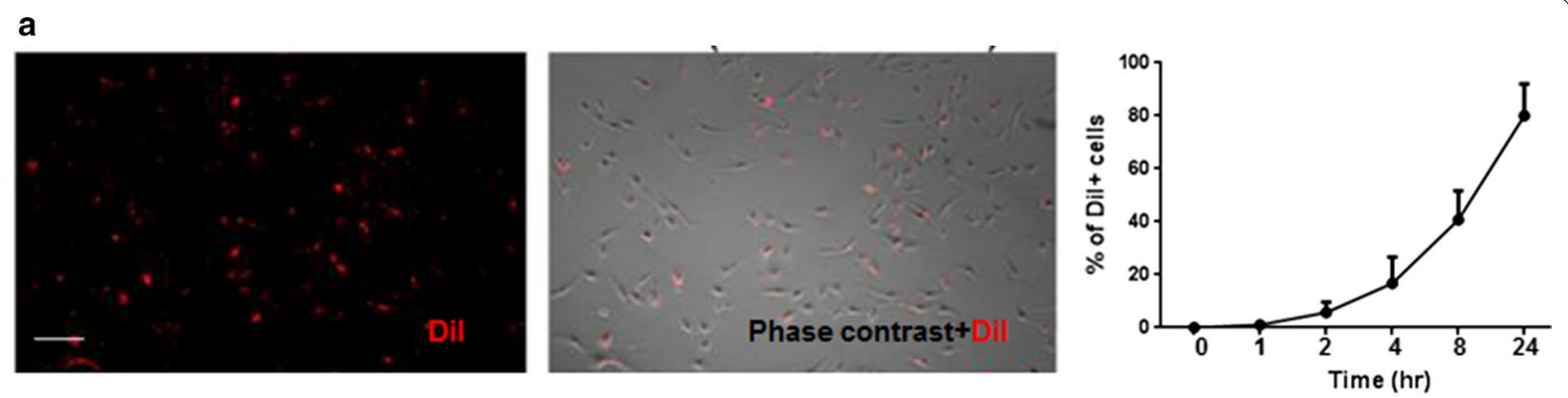

\section{b Naked FITC-UCA1}

\section{Fed: Dil-labeled exosomes containing FITC-UCA1}
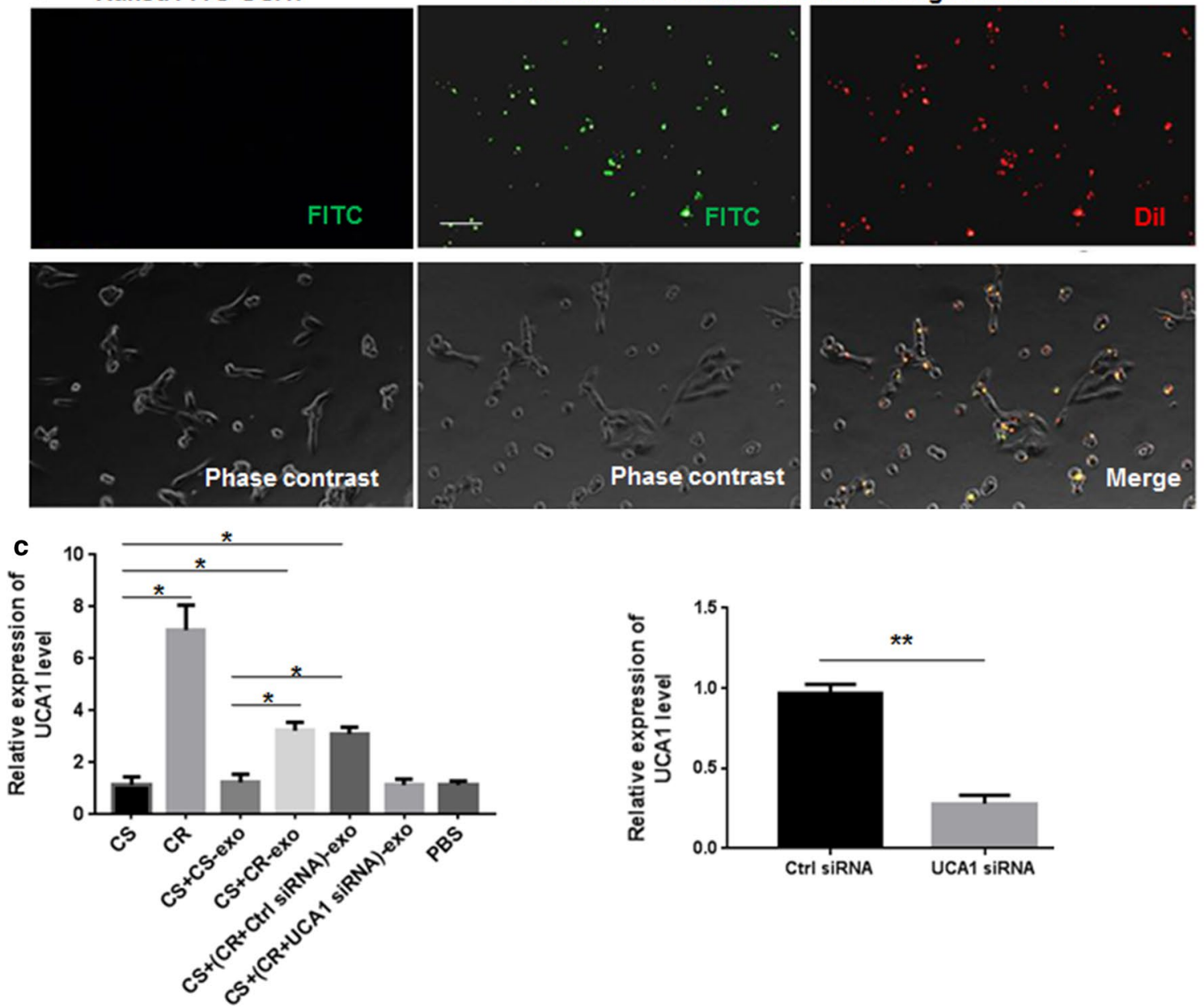

Fig. 4 Intercellular transfer of UCA1 by exosomes. a Left: fluorescence images of Caco2-CS cells incubated with Dil-labeled (red) exosomes derived from Caco2-CR cells for 48 h. Right: flow cytometry analysis of Dil-positive Caco2-CS cells after incubation with Dil-labeled exosomes for the indicated time. $\mathbf{b}$ Fluorescence images of Caco2-CS cells $48 \mathrm{~h}$ after incubation with Dil-labeled (red) exosomes derived from Caco2-CR cells electroporated with FITC-UCA1 (green) or incubated with naked FITC-UCA1. Representative fluorescence images and phase contrast images are shown. Scale bar, 500 mm. c Left: qRT-PCR analysis of UCA1 in Caco2-CS cells (CS) $48 \mathrm{~h}$ after incubation with the indicated exosomes. PBS was used as the control group $(n=3)$. Caco2-CR (CR) cells were used as a positive control. Right: qRT-PCR analysis of UCA1 in UCA1-knockdown and control Caco2-CR cells $(n=3)$

was nearly equal in the $\mathrm{PD} / \mathrm{SD}$ and $\mathrm{PR} / \mathrm{CR}$ groups, interestingly, UCA1 level was markedly higher in $\mathrm{PD} / \mathrm{SD}$ patients than in PR/CR patients. As exosomes are important players in intercellular communication processes
[34-36], we further investigated whether exosomes are involved in drug resistance process and evaluated the significance of the transfer of drug-resistance properties in CRC. We observed that exosomes derived from 

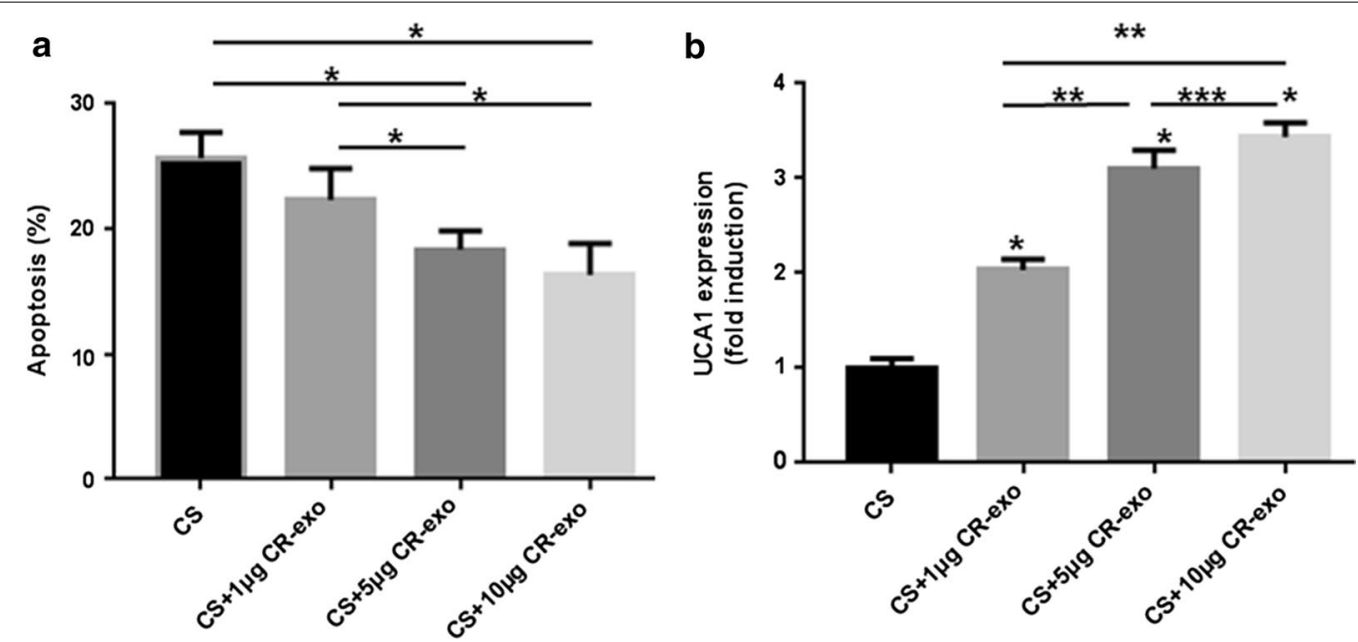

Fig. 5 Exosomes derived from cetuximab-resistant cells conferred drug resistance to sensitive cells and altered UCA1 expression. Caco2-CS cells were treated with cetuximab for $48 \mathrm{~h}$ before flow cytometry analysis after coculture with or without CR-exo for $24 \mathrm{~h}$. The apoptotic rates of Caco2-CS cells exposed to cetuximab were analyzed in four samples: Caco2-CS cells (CS), Caco2-CS cells coincubated with $1 \mu \mathrm{g}$ CR-exo (CS + $1 \mu \mathrm{g}$ CR-exo), Caco2-CS cells coincubated with $5 \mu \mathrm{g}$ CR-exo (CS + $5 \mu \mathrm{g}$ CR-exo), and Caco2-CS cells coincubated with $10 \mu \mathrm{g}$ CR-exo (CS + $10 \mu \mathrm{g}$ CR-exo). a The results are representative of three independent experiments (the mean apoptotic rate \pm SD). Apoptotic rates in the CS $+10 \mu \mathrm{g} C R$-exo and $\mathrm{CS}+5 \mu \mathrm{g}$ CR-exo groups were significantly increased compared with those in the CS and CS $+1 \mu \mathrm{g}$ CR-exo groups $\left({ }^{*} \mathrm{p}<0.05\right)$, while the difference between the CS $+5 \mu \mathrm{g}$ CR-exo and CS $+10 \mu \mathrm{g}$ CR-exo groups was not significant. $\mathbf{b}$ The UCA1 expression level significantly increased in a dose-dependent manner in the above four groups of CR-exo-treated cells: groups incubated with greater amounts of CR-exo showed higher UCA1 expression. Different symbols indicated the significant differences $(p<0.05)\left({ }^{*}\right.$ Compared with $C S ;{ }^{* *}$ Compared with $C S+1 \mu \mathrm{C}$ CR-exo; ${ }^{* * *}$ Compared with $\mathrm{CS}+5 \mu \mathrm{g}$ (R-exo)

cetuximab-resistant cells can transmit drug resistance to sensitive cells and cause changes in UCA1 level. These findings further support for the hypothesis that analyzing exosomal lncRNA expression in blood as a biomarker become ever more feasible and that UCA1-containing exosomes can predict cetuximab therapy efficacy in CRC patients.

The molecular mechanisms by which most lncRNAs function remain to be elucidated. LncRNAs play roles in signaling and can act as decoys, guides or scaffolds by specifically binding to target DNAs, RNAs and proteins. While these mechanisms are not mutually exclusive, some lncRNAs may have one or more roles. In different cancer cells, UCA1 has been demonstrated to bind to several miRNAs [24, 37-43]. In addition to its oncogenic effect, UCA1 also exerts a regulatory effect on drug resistance in several types of cancer. For example, UCA1 can induce acquired resistance to EGFR-tyrosine kinase inhibitors (TKIs) in EGFR-mutant nonsmall cell lung cancer by activating the AKT/mTOR pathway [44]. It can also increase the chemoresistance of bladder cancer cells by activating the Wnt signaling pathway in a Wnt6-dependent manner [45]. Knockdown of UCA1 in adriamycin-resistant SGC7901/ADR cells can significantly decrease resistance [46]. The major limitation of this study is that downstream regulation of UCA1 in cetuximab-resistant cells was not investigated. We hypothesized that some molecular effectors, such as miRNA, might be important targets of UCA1, leading to dysregulation of the signaling pathway in cetuximab resistance. However, further studies are required to validate our hypothesis.

\section{Conclusions}

In summary, this is the first demonstration that exosomal UCA1 has the key characteristics of a tumor marker: it can be assayed in a noninvasive manner, and it is relatively abundant and stable. Moreover, we explored exosomal UCA1 as a biomarker for predicting drug sensitivity in patients who were treated with cetuximab. UCA1-containing exosomes appear to be essential for conferring cetuximab resistance. Although some factors involving in drug resistance has been widely studied, our findings on UCA1 open important possibilities for future clinical application. Thus, both basic and clinical researchers should engage in multidisciplinary efforts monitoring of cetuximab resistance. As mentioned above, UCA1 is a potential good broad-spectrum biomarker for cancer diagnosis, prognosis or prediction of therapeutic response. However, no UCA1 products have been identified in clinical trials. Further preclinical and clinical studies will highlight and better elucidate the role of UCA1 in anticancer drug resistance and may lead to future investigations of the various clinical applications of UCA1. 


\section{Abbreviations}

CRC: colorectal cancer; EGFR: epidermal growth factor receptor; MAb: monoclonal antibody; mCRC: metastatic CRC; IncRNAs: long noncoding RNAs; UCA1: urothelial carcinoma associated 1; PR: partial response; CR: complete response; PD: progressive disease; SD: stable disease; DMEM: Dulbecco's Modified Eagle's Medium; CCK8: Cell Counting Kit 8; PBS: phosphate-buffered saline; TEM: transmission electron microscopy; qRT-PCR: quantitative real-time reverse transcription-polymerase chain reaction; SDS: sodium dodecyl sulfate; ECL: enhanced chemiluminescence; SiRNA: small interfering RNA; PI: propidium iodide; SD: standard deviation; FITC: fluorescein isothiocyanate.

\section{Authors' contributions}

$\mathrm{SJ}$ and $\mathrm{YH}$ designed the experiments and edited the manuscript. $Y Y$ and $\mathrm{RZ}$ conducted the experiments and wrote the manuscript. JD, HY, YL, XW and XD performed experiments and analysed data. All authors read and approved the final manuscript.

\begin{abstract}
Author details
${ }^{1}$ Department of Thoracic Surgery, Harbin Medical University Cancer Hospital, Harbin, Heilongjiang, China. ${ }^{2}$ Department of Colorectal Surgery, Cancer Hospital of China Medical University, Liaoning Cancer Hospital \& Institute, Shenyang, Liaoning, China. ${ }^{3}$ Department of Gastrointestinal Oncology, Harbin Medical University Cancer Hospital, 150 \# Haping Road, Harbin 150081, Heilongjiang, China. ${ }^{4}$ Department of Cardiovascular Surgery, The Second Affiliated Hospital of Harbin Medical University, 246 \# Xuefu Road, Harbin 150001 Heilongjiang, China.
\end{abstract}

\section{Acknowledgements}

Not applicable.

\section{Competing interests}

The authors declare that they have no competing interests.

\section{Availability of data and materials}

All data generated or analysed during this study are included in this published article.

\section{Consent for publication}

All authors agreed on the manuscript.

\section{Ethics approval and consent to participate}

Written informed consent for the biological studies was obtained from each patient involved in the study, and the study was approved by the Ethics Committee of Harbin Medical University Cancer Hospital.

\section{Funding}

This study was supported by the Key Project of Haiyan Research Fund of Harbin Medical University Cancer Hospital (No. JJZD2018-06) and WU Jieping Medical Foundation (No. 320.6750.16172).

\section{Publisher's Note}

Springer Nature remains neutral with regard to jurisdictional claims in published maps and institutional affiliations.

\section{Received: 16 July 2018 Accepted: 8 October 2018}

Published online: 22 October 2018

\section{References}

1. Siegel RL, Miller KD, Jemal A. Cancer statistics, 2016. CA Cancer J Clin. 2016;66(1):7-30.

2. Ye LC, Liu TS, Ren L, Wei Y, Zhu DX, Zai SY, et al. Randomized controlled trial of cetuximab plus chemotherapy for patients with KRAS wildtype unresectable colorectal liver-limited metastases. J Clin Oncol. 2013;31(16):1931-8

3. Amado RG, Wolf M, Peeters M, Van Cutsem E, Siena S, Freeman DJ, et al. Wild-type KRAS is required for panitumumab efficacy in patients with metastatic colorectal cancer. J Clin Oncol. 2008;26(10):1626-34.
4. Maughan TS, Adams RA, Smith CG, Meade AM, Seymour MT, Wilson RH, et al. Addition of cetuximab to oxaliplatin-based first-line combination chemotherapy for treatment of advanced colorectal cancer: results of the randomised phase 3 MRC COIN trial. Lancet. 2011;377(9783):2103-14.

5. Di Nicolantonio F, Martini M, Molinari F, Sartore-Bianchi A, Arena S, Saletti $P$, et al. Wild-type BRAF is required for response to panitumumab or cetuximab in metastatic colorectal cancer. J Clin Oncol. 2008;26(35):5705-12.

6. De Roock W, Claes B, Bernasconi D, De Schutter J, Biesmans B, Fountzilas $G$, et al. Effects of KRAS, BRAF, NRAS, and PIK3CA mutations on the efficacy of cetuximab plus chemotherapy in chemotherapy-refractory metastatic colorectal cancer: a retrospective consortium analysis. Lancet Oncol. 2010;11(8):753-62.

7. Bertotti A, Migliardi G, Galimi F, Sassi F, Torti D, Isella C, et al. A molecularly annotated platform of patient-derived xenografts ("xenopatients") identifies HER2 as an effective therapeutic target in cetuximab-resistant colorectal cancer. Cancer Discov. 2011;1(6):508-23.

8. Bardelli A, Corso S, Bertotti A, Hobor S, Valtorta E, Siravegna G, et al. Amplification of the MET receptor drives resistance to anti-EGFR therapies in colorectal cancer. Cancer Discov. 2013:3(6):658-73.

9. Qi P, Du X.The long non-coding RNAs, a new cancer diagnostic and therapeutic gold mine. Mod Pathol. 2013;26(2):155-65.

10. Mercer TR, Dinger ME, Mattick JS. Long non-coding RNAs: insights into functions. Nat Rev Genet. 2009;10(3):155-9.

11. Prensner JR, Chinnaiyan AM. The emergence of IncRNAs in cancer biology. Cancer Discov. 2011;1(5):391-407.

12. Qu L, Ding J, Chen C, Wu ZJ, Liu B, Gao Y, et al. Exosome-transmitted InCARSR promotes sunitinib resistance in renal cancer by acting as a competing endogenous RNA. Cancer Cell. 2016;29(5):653-68.

13. Qi P, Xu MD, Ni SJ, Huang D, Wei P, Tan C, et al. Low expression of LOC285194 is associated with poor prognosis in colorectal cancer. J Trans Med. 2013;11:122.

14. Qi P, Xu MD, Ni SJ, Shen XH, Wei P, Huang D, et al. Down-regulation of ncRAN, a long non-coding RNA, contributes to colorectal cancer cell migration and invasion and predicts poor overall survival for colorectal cancer patients. Mol Carcinog. 2015;54(9):742-50.

15. Xue M, Chen W, Li X. Urothelial cancer associated 1: a long noncoding RNA with a crucial role in cancer. J Cancer Res Clin Oncol. 2016;142(7):1407-19.

16. Han Y, Yang YN, Yuan HH, Zhang TT, Sui H, Wei XL, et al. UCA1, a long noncoding RNA up-regulated in colorectal cancer influences cell proliferation, apoptosis and cell cycle distribution. Pathology. 2014;46(5):396-401.

17. Wang H, Guan Z, He K, Qian J, Cao J, Teng L. LncRNA UCA1 in anti-cancer drug resistance. Oncotarget. 2017;8(38):64638-50.

18. Dong L, Lin W, Qi P, Xu MD, Wu X, Ni S, et al. Circulating long RNAs in serum extracellular vesicles: their characterization and potential application as biomarkers for diagnosis of colorectal cancer. Cancer Epidemiol Biomark Prev. 2016;25(7):1158-66

19. Qi P, Zhou XY, Du X. Circulating long non-coding RNAs in cancer: current status and future perspectives. Mol Cancer. 2016;15(1):39.

20. Wang J, Liu Y, Sun W, Zhang Q, Gu T, Li G. Plasma exosomes as novel biomarker for the early diagnosis of gastric cancer. Cancer Biomark. 2018;21(4):805-12.

21. Tian Y, Li S, Song J, Ji T, Zhu M, Anderson GJ, et al. A doxorubicin delivery platform using engineered natural membrane vesicle exosomes for targeted tumor therapy. Biomaterials. 2014;35(7):2383-90.

22. Melo SA, Sugimoto H, O'Connell JT, Kato N, Villanueva A, Vidal A, et al. Cancer exosomes perform cell-independent microRNA biogenesis and promote tumorigenesis. Cancer Cell. 2014;26(5):707-21.

23. Peinado H, Alečković M, Lavotshkin S, Matei I, Costa-Silva B, MorenoBueno $\mathrm{G}$, et al. Melanoma exosomes educate bone marrow progenitor cells toward a pro-metastatic phenotype through MET. Nat Med. 2012;18(6):883-91.

24. Bian Z, Jin L, Zhang J, Yin Y, Quan C, Hu Y, et al. LncRNA-UCA1 enhances cell proliferation and 5-fluorouracil resistance in colorectal cancer by inhibiting miR-204-5p. Sci Rep. 2016;6:23892.

25. Fan $Q$, Yang $L$, Zhang X, Peng X, Wei S, Su D, et al. The emerging role of exosome-derived non-coding RNAs in cancer biology. Cancer Lett. 2018:414:107-15

26. Bobrie A, Krumeich S, Reyal F, Recchi C, Moita LF, Seabra MC, et al. Rab27a supports exosome-dependent and -independent mechanisms that 
modify the tumor microenvironment and can promote tumor progression. Cancer Res. 2012;72(19):4920-30.

27. Webber JP, Spary LK, Sanders AJ, Chowdhury R, Jiang WG, Steadman R, et al. Differentiation of tumour-promoting stromal myofibroblasts by cancer exosomes. Oncogene. 2015;34(3):290-302.

28. Wang J, Hendrix A, Hernot S, Lemaire M, De Bruyne E, Van Valckenborgh E, et al. Bone marrow stromal cell-derived exosomes as communicators in drug resistance in multiple myeloma cells. Blood. 2014;124(4):555-66.

29. Shao H, Chung J, Lee K, Balaj L, Min C, Carter BS, et al. Chip-based analysis of exosomal mRNA mediating drug resistance in glioblastoma. Nat Commun. 2015;6:6999.

30. Zhang L, Pan L, Xiang B, Zhu H, Wu Y, Chen M, et al. Potential role of exosome-associated microRNA panels and in vivo environment to predict drug resistance for patients with multiple myeloma. Oncotarget. 2016;7(21):30876-91.

31. Chen Y, Xie Y, Xu L, Zhan S, Xiao Y, Gao Y, et al. Protein content and functional characteristics of serum-purified exosomes from patients with colorectal cancer revealed by quantitative proteomics. Int J Cancer. 2017;140(4):900-13.

32. Sugimachi $K$, Matsumura T, Hirata H, Uchi R, Ueda M, Ueo H, et al. Identification of a bona fide microRNA biomarker in serum exosomes that predicts hepatocellular carcinoma recurrence after liver transplantation. Br J Cancer. 2015;112(3):532-8.

33. Ma X, Chen Z, Hua D, He D, Wang L, Zhang P, et al. Essential role for TrpC5containing extracellular vesicles in breast cancer with chemotherapeutic resistance. Proc Natl Acad Sci USA. 2014;111(17):6389-94.

34. Kong JN, He Q, Wang G, Dasgupta S, Dinkins MB, Zhu G, et al. Guggulsterone and bexarotene induce secretion of exosome-associated breast cancer resistance protein and reduce doxorubicin resistance in MDA-MB-231 cells. Int J Cancer. 2015;137(7):1610-20.

35. Wang J, De Veirman K, De Beule N, Maes K, De Bruyne E, Van Valckenborgh $E$, et al. The bone marrow microenvironment enhances multiple myeloma progression by exosome-mediated activation of myeloidderived suppressor cells. Oncotarget. 2015;6(41):43992-4004.

36. Zhang L, Zhang S, Yao J, Lowery FJ, Zhang Q, Huang WC, et al. Microenvironment-induced PTEN loss by exosomal microRNA primes brain metastasis outgrowth. Nature. 2015;527(7576):100-4.
37. Wang F, Ying HQ, He BS, Pan YQ, Deng QW, Sun HL, et al. Upregulated IncRNA-UCA1 contributes to progression of hepatocellular carcinoma through inhibition of miR-216b and activation of FGFR1/ERK signaling pathway. Oncotarget. 2015;6(10):7899-917.

38. Nie W, Ge HJ, Yang XQ, Sun $X$, Huang H, Tao X, et al. LncRNA-UCA1 exerts oncogenic functions in non-small cell lung cancer by targeting miR193a-3p. Cancer Lett. 2016;371(1):99-106.

39. Xue M, Pang H, Li X, Li H, Pan J, Chen W. Long non-coding RNA urothelial cancer-associated 1 promotes bladder cancer cell migration and invasion by way of the hsa-miR-145-ZEB1/2-FSCN1 pathway. Cancer Sci. 2016;107(1):18-27.

40. Jiao C, Song Z, Chen J, Zhong J, Cai W, Tian S, et al. IncRNA-UCA1 enhances cell proliferation through functioning as a ceRNA of Sox 4 in esophageal cancer. Oncol Rep. 2016;36(5):2960-6.

41. Wang T, Yuan J, Feng N, Li Y, Lin Z, Jiang Z, et al. Hsa-miR-1 downregulates long non-coding RNA urothelial cancer associated 1 in bladder cancer. Tumour Biol. 2014;35(10):10075-84.

42. Li X, Wu Y, Liu A, Tang X. Long non-coding RNA UCA1 enhances tamoxifen resistance in breast cancer cells through a miR-18a-HIF1 a feedback regulatory loop. Tumour Biol. 2016;37(11):14733-43.

43. Xiao Y, Jiao C, Lin Y, Chen M, Zhang J, Wang J, et al. IncRNA UCA1 contributes to imatinib resistance by acting as a ceRNA against miR-16 in chronic myeloid leukemia cells. DNA Cell Biol. 2017;36(1):18-25.

44. Cheng N, Cai W, Ren S, Li X, Wang Q, Pan H, et al. Long non-coding RNA UCA1 induces non-T790M acquired resistance to EGFR-TKIs by activating the AKT/mTOR pathway in EGFR-mutant non-small cell lung cancer. Oncotarget. 2015;6(27):23582-93.

45. Fan Y, Shen B, Tan M, Mu X, Qin Y, Zhang F, et al. Long non-coding RNA UCA1 increases chemoresistance of bladder cancer cells by regulating Wnt signaling. FEBS J. 2014;281(7):1750-8.

46. Shang C, Guo Y, Zhang J, Huang B. Silence of long noncoding RNA UCA1 inhibits malignant proliferation and chemotherapy resistance to adriamycin in gastric cancer. Cancer Chemother Pharmacol. 2016;77(5):1061-7.
Ready to submit your research? Choose BMC and benefit from:

- fast, convenient online submission

- thorough peer review by experienced researchers in your field

- rapid publication on acceptance

- support for research data, including large and complex data types

- gold Open Access which fosters wider collaboration and increased citations

- maximum visibility for your research: over 100M website views per year

At BMC, research is always in progress.

Learn more biomedcentral.com/submissions 